\title{
Violência na infância, exposição a violência parental e abuso e/ou dependência de álcool na idade adulta*
}

\author{
Daniela Viganó Zanoti-Jeronymo ${ }^{1}$ \\ Marcos José Barreto Zaleski \\ llana Pinsky ${ }^{3}$ \\ Raul Caetano 4 \\ Neliana Buzi Figlie ${ }^{3}$ \\ Ronaldo Ramos Laranjeira ${ }^{3}$
}

Este artigo examina a associação entre o histórico de violência na infância e a dependência e uso nocivo de álcool na idade adulta. Uma amostra probabilística multiestagio foi usada para selecionar 3.007 indivíduos de 14 anos de idade ou mais, dos lares brasileiros, entre novembro de 2005 e abril de 2006. As análises mostraram a existência de associação entre o histórico de abuso físico e exposição à violência parental $(p<0,001)$. O modelo de regressão multinomial mostrou uma associação entre o histórico de violência na infância e a dependência de álcool na idade adulta. As variáveis de maior impacto na chance para dependência do álcool foram sexo masculino, exposição à violência parental e histórico de abuso físico. Desta forma, observou-se que quanto maior o grau de exposição, maior a porcentagem de violência severa sofrida na infância. Os resultados corroboram com prévios achados que sugerem que um histórico positivo de violência na infância é um componente importante na etiologia dos transtornos decorrentes do consumo de álcool.

Descritores: Violência Doméstica; Abuso na Infância; Violência Parental; Dependência e Abuso de Álcool; Estudos Transversais.

\footnotetext{
Artigo extraído da tese de doutorado "Associação entre história de violência sofrida na infância e transtornos relacionados ao uso de álcool na idade adulta", apresentada à Universidade Federal de São Paulo, Escola Paulista de Medicina, São Paulo, SP, Brasil.

${ }^{1}$ Universidade Estadual do Centro Oeste, Centro de Ciências da Saúde, Guarapuava, PR, Brasil.

2 Universidade Federal de Santa Catarina, Centro de Ciências da Saúde, Florianópolis, SC, Brasil.

${ }^{3}$ Universidade Federal de São Paulo, Escola Paulista de Medicina, São Paulo, SP, Brasil.

${ }^{4}$ University of Texas, School of Public Health, Dallas, TX, Estados Unidos da América.
} 


\section{Childhood violence, exposure to parental violence and alcohol dependence/abuse in adulthood}

This article analyses the association between the history of violence in childhood and alcohol abuse and dependence in adulthood. A multistage probabilistic sample was used to select 3,007 individuals aged 14 years or older, living in Brazil, between November 2005 and April 2006. Analyses showed an association between history of physical abuse and exposure to parental violence $(p<0.001)$. A multinomial regression model showed a significant association between history of physical abuse in childhood and alcohol dependence in adulthood. The following variables have greater impact on the probability of alcohol dependence: male sex, exposure to parental violence and history of physical abuse. Thus, the higher the level of exposure, the greater the percentage of severe violence suffered in childhood. Results corroborate previous findings, which suggest the importance of a childhood history of violence in the etiology of disorders resulting from alcohol consumption.

Descriptors: Domestic Violence; Abuse in Childhood; Parental Violence; Alcohol Dependence and Abuse; Cross-Sectional Studies.

\section{La violencia infantil, exposición a la violencia de los padres y el abuso y/o dependencia del alcohol en la edad adulta}

En este artículo se estudia la relación entre la historia de la violencia en la infancia y la dependencia y el uso nocivo de alcohol en la edad adulta. Una muestra de probabilidad de varias etapas fue utilizada para seleccionar 3.007 individuos de 14 años de edad o más de los hogares brasileños entre noviembre de 2005 y abril de 2006. El análisis mostró que hay una asociación entre la historia de abuso físico y la exposición a la violencia de los padres $(p<0,001)$. El modelo de regresión multinomial mostró una asociación entre la historia de violencia en la infancia y la dependencia del alcohol en la edad adulta. Las variables con mayor impacto en la probabilidad de dependencia del alcohol fueran el sexo masculino, la exposición a la violencia de los padres y la historia de abuso físico. Cuanto mayor sea el grado de exposición, mayor es el porcentaje de violencia grave sufrido en la infancia. Los resultados corroboran hallazgos previos que sugieren que una historia positiva de violencia en la infancia es un componente importante en la etiología de los trastornos derivados del consumo de alcohol.

Descriptores: Violencia Doméstica; Abuso en la Infancia; Violencia de los Padres; Adicción y Abuso de Alcohol; Estudios Transversales.

Introdução

Por sua elevada incidência e prevalência, a violência doméstica é reconhecida mundialmente como um importante problema de saúde pública ${ }^{(1-2)}$, e suas consequências na saúde física e mental impactam na vida adulta(3-4). 
Evidências de várias pesquisas sustentam que a vitimização na infância através do abuso físico e da observação da violência parental entre os pais pode conduzir a um risco elevado de desenvolver problemas de saúde física ${ }^{(5-6)}$ e altas taxas de doenças psiquiátricas na idade adulta, tais como depressão, ansiedade e abuso de substâncias ${ }^{(3,6-7)}$.

Com relação ao uso de substâncias, especificamente o abuso e/ou dependência de álcool, estudos prévios apontam experiências de violência na infância como um fator de risco para o desenvolvimento de transtornos relacionados ao álcool(8), enquanto outros são mais incisivos, e relatam que tais experiências são um componente importante na etiologia de desordens de uso de álcool(9-10).

Evidências comprovam que abuso físico e exposição à violência parental tendem a coocorrer, o que indica que estas experiências não são eventos isolados na vida dos sujeitos com história de violência na infância(1,11-12). A acumulação destes dois tipos de experiências, abuso físico e exposição à violência parental, pode gerar consequências negativas em curto e a longo prazo e levar a uma maior vulnerabilidade para o desenvolvimento de problemas comportamentais $\mathbf{s}^{(5,11,13)}$.

Esta associação tem sido relatada em diversos estudos realizados em diferentes países ${ }^{(9-10,14-15)}$. Entretanto, comparada a estudos realizados nos países desenvolvidos, a produção científica brasileira sobre a verificação da associação de história de violência na infância e desordens de uso de álcool na idade adulta é pouco estudada. Assim, o objetivo deste estudo foi avaliar os efeitos do abuso físico e da exposição à violência parental na infância sobre o risco de desenvolver dependência e abuso do álcool na idade adulta em uma amostra representativa da população brasileira.

\section{Método}

\section{Procedimento de amostragem e coleta de dados}

O estudo atual analisa dados vindos do I levantamento nacional sobre os padrões de consumo de álcool na população brasileira ${ }^{(16)}$, conduzido pela Unidade de Pesquisa em Álcool e Drogas (UNIAD) da Universidade Federal de São Paulo (Unifesp). Os dados foram coletados entre novembro 2005 e abril 2006 em 143 cidades brasileiras, totalizando 325 setores censitários e utilizando a metodologia de amostra probabilística estratificada em três estágios. Estágio 1: sorteio sistemático dos municípios selecionados probabilisticamente pelo método PPT (Probabilidade
Proporcional ao Tamanho), sendo a população de cada município tomada como base para tal seleção. Estágio 2: seleção de dois setores censitários para cada cidade, com exceção das 14 maiores cidades selecionadas, totalizando 325 setores censitários, e usando também o PPT. Estágio 3: dentro de cada setor, foram selecionadas oito casas por amostragem aleatória simples, seguida pela seleção de um membro da casa a ser entrevistado usando "a técnica do próximo aniversariante", a contar da data do arrolamento do domicílio.

A metodologia de amostragem foi desenhada para ter abrangência de todo o território nacional, representando toda a população brasileira de 14 anos de idade ou mais, de ambos os sexos, e sem exclusão de qualquer parte do território nacional, inclusive áreas rurais. Populações especiais como índios brasileiros, militares e institucionalizados não foram incluídas na amostra.

A amostra foi composta por 2.522 entrevistas com participantes de 14 anos de idade ou mais e uma cota extra de 485 entrevistas, esta última exclusiva para a população entre 14 e 17 anos de idade, totalizando 3.007 entrevistas. Uma hora de entrevista face a face foi conduzida na casa dos respondentes por entrevistadores treinados que usaram um questionário fechado e padronizado. A taxa de resposta foi de $66.4 \%$. $A$ recusa foi mais elevada entre sujeitos de status socioeconômico elevado.

Todos os respondentes concederam seu consentimento livre e esclarecido para o estudo original que foi aprovado pelo Comitê de ética em pesquisa da Universidade Federal de São Paulo.

\section{Mensurações}

O instrumento utilizado para coleta de dados do I Levantamento Nacional sobre os Padrões de Consumo de Álcool na população brasileira foi uma versão do questionário utilizado no Hispanic Americans Baseline Alcohol Survey (HABLAS) ${ }^{(17)}$. O questionário foi traduzido pelos coordenadores da pesquisa e passou por um processo de adaptação à realidade sociocultural da população brasileira.

- Histórico de violência: Os itens do HABLAS para avaliação do abuso físico na infância foram adaptados da Conflict Tactics Scales (CTS), Form $\mathrm{R}^{(18)}$, e Revised Conflict Tactics Scales (CTS2)(19). Desta forma, neste trabalho, o termo "abuso" foi restrito a abuso físico intrafamiliar, caracterizado pelo uso da força física de pais e/ou cuidadores contra crianças e adolescentes e violência parental, também caracterizada pelo uso da força física com a intenção de provocar danos ao 
parceiro íntimo, de acordo com os critérios de Straus \& Gelles ${ }^{(20)}$

Abuso físico na infância: Os respondentes foram questionados se em alguma ocasião durante sua infância e/ou adolescência seus pais ou as pessoas que os criaram bateram com alguma coisa, bateram em você, queimaram ou escaldaram, ameaçaram com faca ou arma, ou usaram faca ou arma. De acordo com suas respostas, os indivíduos foram alocados em um dos três grupos, a saber: sem história de abuso físico na infância, abuso físico moderado (bateram em você), ou abuso físico severo na infância (bateu em você com alguma coisa, queimou ou escaldou, ameaçou com uma faca ou uma arma, ou usou uma faca ou uma arma).

Exposição à violência parental na infância: Os respondentes foram questionados se durante sua infância ou adolescência observaram seus pais ou as pessoas que os criaram ameaçarem um ao outro de praticarem violência física, ou serem realmente fisicamente violentos um com o outro. Com base nas suas respostas, os indivíduos foram alocados em três grupos, a saber: sem exposição à violência parental (nunca observaram ameaça de violência ou violência física parental), exposição à violência parental moderada (testemunharam ameaça de agressão parental) e exposição à violência parental severa (testemunharam ameaça e agressão física parental juntas).

- Dependência e abuso de álcool: Os dados para verificar o abuso ou a dependência de álcool foram obtidos através de questões do Composite International Diagnostic Interview-Substance (CIDISAM) ${ }^{(21)}$ (REF). Para avaliação do tempo de ocorrência foram investigados os sintomas presentes nos últimos 12 meses. Dessa forma, entendeu-se como: Abuso: Resposta positiva a pelo menos uma das seis perguntas sobre o abuso de álcool do CIDI; Dependência: Resposta positiva a pelo menos três das sete perguntas sobre dependência do CIDI.

- Variáveis sociodemográficas: As variáveis usadas foram: gênero; idade; raça; estado civil; nível educacional; renda familiar; situação de trabalho; regiões geográficas Brasil.

- Análise estatística: As análises foram realizadas através do programa SPSS 13.0. Realizaram-se análises descritivas para caracterização da amostra e da distribuição do histórico de violência na infância e desordens de uso de álcool. Em seguida, para se estabelecer associação entre ocorrência de história de abuso físico e exposição à violência parental na infância com diagnóstico de abuso ou dependência de álcool, foram utilizados testes qui-quadrado de Pearson. Foi utilizado um modelo de regressão multinomial.
Inicialmente, foram incluídas as variáveis: sexo, idade, estado civil, renda familiar, região, raça, situação de trabalho, exposição à violência na infância e histórico de violência na infância como variáveis explicativas. As variáveis não significantes a um nível de $5 \%$ foram excluídas do modelo uma a uma (método backward). Para todas as análises, a estrutura complexa do plano amostral via módulo "complex samples" do SPSS foi levada em consideração.

Considerações Éticas: O estudo foi aprovado pelo Comitê de Ética em Pesquisa da Universidade Federal de São Paulo (código: CEP 1672/04). Todos os entrevistados assinaram o termo de consentimento livre e esclarecido e tiveram certeza da natureza confidencial do estudo antes da entrevista.

\section{Resultados}

\section{Características da amostra estudada}

A população do estudo incluiu 1.285 homens $(47,9 \%)$ e 1722 mulheres $(52,10 \%)$. A idade média foi de 36,84 anos para os homens e 38,01 anos para as mulheres (variando de 14 a 91 anos). Metade dos sujeitos era de cor da pele branca (homens $=51,4 \%$, mulheres $=49,7 \%$ ), $35,2 \%$ eram pardos (homens $=$ $34,7 \%$, mulheres $=35,6 \%), 11,3 \%$ eram negros (homens $=10,9 \%$, mulheres $=11,7 \%$ ) e $3 \%$ eram de outras raças. Com relação ao estado civil, $55,4 \%$ eram casados ou viviam com companheiro(a) (homens= $58,2 \%$, mulheres $=52,8 \%$ ). Aproximadamente $34 \%$ dos sujeitos apresentavam baixos níveis de instrução (homens $=34,2 \%$, mulheres $=33,9 \%$ ), sendo que destes, $23 \%$ nunca frequentaram a escola ou tinham apenas as séries iniciais do ensino fundamental completas. Para a variável situação de trabalho, $70,5 \%$ dos sujeitos exerciam algum tipo de atividade remunerada (homens= $83,2 \%$, mulheres $=58,7 \%$ ) e $25 \%$ das mulheres relataram serem donas de casa. Aproximadamente dois quintos da amostra $(39,1 \%)$ relataram uma renda familiar mensal de até $R \$ 450,00$.

A amostra foi contemplada com sujeitos de todas as regiões do país, apresentando representatividade nacional. Estes resultados são também apresentados em Zanoti-Jeronymo ${ }^{(13)}$.

\section{Prevalência de história de violência na infância}

Conforme os dados da Tabela 1, na amostra estudada, $44,1 \%$ dos sujeitos relataram história positiva de abuso físico na infância (homens $=45,5 \%$, mulheres $=42,8 \%$ ). Destes, 33,8\% (homens $=35,8 \%$, mulheres $=32,0 \%$ ) relataram abuso físico moderado e 
$10,3 \%$ (homens $=9,7 \%$, mulheres $=10,8 \%$ ) relataram abuso físico severo. A prevalência de exposição à violência parental na infância foi de $26,1 \%$ (homens= $24,1 \%$, mulheres $=27,9 \%)$, sendo que $7,5 \%$ testemunharam violência parental moderada (homens= $8,3 \%$, mulheres $=6,7 \%$ ) e $18,6 \%$ testemunharam violência parental severa (homens $=15,8 \%$, mulheres= $21,2 \%)$.

Tabela 1 - Prevalência de abuso físico e exposição à violência parental na infância

\begin{tabular}{lccc}
\hline & Homem & Mulher & Total \\
\hline História de violência na infância & $100 \%$ & $100 \%$ & $100 \%$ \\
Nenhum abuso & $54.5 \%$ & $57.1 \%$ & $55.9 \%$ \\
Abuso físico moderado & $35.8 \%$ & $32 \%$ & $33.8 \%$ \\
Abuso físico severo & $9.7 \%$ & $10.8 \%$ & $10.3 \%$ \\
& & $100 \%$ & $73.9 \%$ \\
Exposição à violência parental & $100 \%$ & $72 \%$ & $7.5 \%$ \\
Sem exposição à violência parental & $75.9 \%$ & $6.7 \%$ & $18.60 \%$ \\
Exposição à violência parental moderada & $8.3 \%$ & $21.20 \%$ & $100 \%$ \\
Exposição à violência parental severa & $15.80 \%$ & & \\
\hline
\end{tabular}

Houve uma forte associação entre exposição à violência parental e história de abuso físico na infância (Tabela 2). Assim, nos sujeitos sem história de exposição à violência parental, a porcentagem de indivíduos que sofreram algum tipo de violência ("abuso físico moderado" ou "abuso físico severo") não ultrapassou
$40 \%$, tanto em homens como em mulheres. Já nos sujeitos que sofreram algum tipo de exposição, esta porcentagem foi bem maior (acima de 55\%). Da mesma forma, tanto homens (42,5\%) quanto mulheres $(58,0 \%)$ com história de abuso físico severo tendem a ter sido mais expostos à violência parental severa na infância.

Tabela 2 - Associação entre violência parental e abuso físico na infância

\begin{tabular}{|c|c|c|c|c|c|c|c|c|}
\hline \multirow{3}{*}{ Características } & \multicolumn{8}{|c|}{ Frequência relativa } \\
\hline & \multicolumn{4}{|c|}{ Homem } & \multicolumn{4}{|c|}{ Mulher } \\
\hline & $\begin{array}{l}\text { Nenhum } \\
\text { abuso }\end{array}$ & $\begin{array}{c}\text { Abuso } \\
\text { físico } \\
\text { moderado }\end{array}$ & $\begin{array}{l}\text { Abuso } \\
\text { físico } \\
\text { severo }\end{array}$ & Total & $\begin{array}{l}\text { Nenhum } \\
\text { abuso }\end{array}$ & $\begin{array}{c}\text { Abuso } \\
\text { físico } \\
\text { moderado }\end{array}$ & $\begin{array}{l}\text { Abuso } \\
\text { físico } \\
\text { severo }\end{array}$ & Total \\
\hline $\begin{array}{l}\text { Exposição à violência } \\
\text { parental }\end{array}$ & $54.7 \%$ & $35.5 \%$ & $9.8 \%$ & $100 \%$ & $57.1 \%$ & $32.1 \%$ & $10.9 \%$ & $100 \%$ \\
\hline $\begin{array}{l}\text { Sem exposição à } \\
\text { violência parental }\end{array}$ & $62 \%$ & $33 \%$ & $5.1 \%$ & $100 \%$ & $64.1 \%$ & $30.7 \%$ & $5.1 \%$ & $100 \%$ \\
\hline $\begin{array}{l}\text { Exposição à violência } \\
\text { parental moderada }\end{array}$ & $30.1 \%$ & $48.6 \%$ & $21.3 \%$ & $100 \%$ & $44.4 \%$ & $42.5 \%$ & $13.1 \%$ & $100 \%$ \\
\hline $\begin{array}{l}\text { Exposição à violência } \\
\text { parental severa }\end{array}$ & $32.6 \%$ & $41 \%$ & $26.4 \%$ & $100 \%$ & $37.1 \%$ & $33.3 \%$ & $29.6 \%$ & $100 \%$ \\
\hline
\end{tabular}

${ }^{*} p^{1}<0.0001 ; p^{2}<0.0001$ 
Prevalência de desordens de uso de álcool

A prevalência de dependência de álcool foi de $8,2 \%$ da amostra (homens $=13,1 \%$, mulheres $=3,6 \%$ ). A tendência de apresentar mais dependência de álcool foi maior nos homens do que nas mulheres ( $p<0,0001)$. Para abuso de álcool, foi encontrada uma prevalência de $2,9 \%$ (homens $=4,9 \%$, mulheres $=1 \%$ ).

\section{Associação entre história de violência na infância e dependência e abuso de álcool na idade adulta}

A Tabela 3 mostra a prevalência de transtornos relacionados ao uso de álcool referentes à violência na infância e exposição à violência parental. As análises mostraram maior ocorrência do diagnóstico de dependência de álcool (homens $=21,3 \%$, mulheres $=10,8 \%$ ) entre os indivíduos que tinham histórico de abuso físico na infância em comparação com outras situações (sem dependência ou abuso de álcool). Portanto, a dependência do álcool tornou-se mais prevalente entre os homens de acordo com o nível de violência sofrido na infância: nenhum $(9,6 \%)$, abuso físico moderado (16,3\%) e abuso físico severo $(21,3 \%)$. Em contraste, as entre as mulheres, as prevalências de nenhum abuso (2,9\%) e abuso físico moderado $(2,5 \%)$ foram semelhantes e inferiores às da violência física severa $(10,8 \%)$.

Os homens apresentaram distribuições semelhantes de exposição segundo abuso e dependência de álcool, sem diferença significativa entre os grupos $(p=0,2164)$. Por outro lado, houve maior porcentagem de mulheres com dependência entre aquelas que haviam presenciado violência parental moderada e porcentagem ainda maior entre aquelas que testemunharam violência parental severa (6,7\% e $8,5 \%$, respectivamente).

Tabela 3 - Associação entre exposição à violência parental e abuso físico na infância e o uso nocivo ou dependência de álcool na idade adulta

\begin{tabular}{|c|c|c|c|c|c|c|c|c|}
\hline \multirow[b]{2}{*}{ Características } & \multicolumn{4}{|c|}{ Homem } & \multicolumn{4}{|c|}{ Mulher } \\
\hline & $\begin{array}{c}\text { Sem } \\
\text { abuso e/ou } \\
\text { dependência }\end{array}$ & $\begin{array}{l}\text { Abuso } \\
\text { de } \\
\text { álcool }\end{array}$ & $\begin{array}{l}\text { Dependência } \\
\text { de álcool }\end{array}$ & Total & $\begin{array}{c}\text { Sem } \\
\text { abuso e/ou } \\
\text { dependência }\end{array}$ & $\begin{array}{c}\text { Abuso } \\
\text { de } \\
\text { álcool }\end{array}$ & $\begin{array}{l}\text { Dependência } \\
\text { de álcool }\end{array}$ & Total \\
\hline $\mathrm{N}$ & 1.071 & 62 & 143 & 1.276 & 1.635 & 20 & 62 & 1.717 \\
\hline $\begin{array}{l}\text { Exposição } \\
\text { à violência } \\
\text { parental }\end{array}$ & $82.2 \%$ & $4.9 \%$ & $12.9 \%$ & $100 \%$ & $95.4 \%$ & $1.0 \%$ & $3.6 \%$ & $100 \%$ \\
\hline $\begin{array}{l}\text { Sem exposição à } \\
\text { violência parental }\end{array}$ & $83.5 \%$ & $4.6 \%$ & $11.9 \%$ & $100 \%$ & $97 \%$ & $1.1 \%$ & $1.9 \%$ & $100 \%$ \\
\hline $\begin{array}{l}\text { Exposição à } \\
\text { violência parental } \\
\text { moderada }\end{array}$ & $82.1 \%$ & $6.9 \%$ & $11 \%$ & $100 \%$ & $93.3 \%$ & & $6.7 \%$ & $100 \%$ \\
\hline $\begin{array}{l}\text { Exposição à } \\
\text { violência parental } \\
\text { severa }\end{array}$ & $75.8 \%$ & $5.4 \%$ & $18.8 \%$ & $100 \%$ & 90.70 & $0.7 \%$ & $8.5 \%$ & $100 \%$ \\
\hline $\mathrm{p}^{1}=0.2164$ & & & & & & & & \\
\hline $\mathrm{p}^{2}<0.0001$ & & & & & & & & \\
\hline $\begin{array}{l}\text { História de } \\
\text { violência na } \\
\text { infância }\end{array}$ & $82 \%$ & $4.9 \%$ & $13.1 \%$ & $100 \%$ & $95.4 \%$ & $1.0 \%$ & $3.6 \%$ & $100 \%$ \\
\hline Nenhum abuso & $85 \%$ & $5.4 \%$ & $9.6 \%$ & $100 \%$ & $96.2 \%$ & $1.0 \%$ & $2.9 \%$ & $100 \%$ \\
\hline $\begin{array}{l}\text { Abuso físico } \\
\text { moderado }\end{array}$ & $79 \%$ & $4.8 \%$ & $16.3 \%$ & $100 \%$ & $96.5 \%$ & $1.0 \%$ & $2.5 \%$ & $100 \%$ \\
\hline $\begin{array}{l}\text { Abuso físico } \\
\text { severo }\end{array}$ & $75.8 \%$ & $3 \%$ & $21.3 \%$ & $100 \%$ & $88 \%$ & $1.1 \%$ & $10.8 \%$ & $100 \%$ \\
\hline$p^{1}=0.0063$ & & & & & & & & \\
\hline$p^{2}=0.0005$ & & & & & & & & \\
\hline
\end{tabular}




\section{Resultados da regressão multinomial}

A Tabela 4 mostra as variáveis que permaneceram no modelo final da regressão multinomial. A análise dos resultados quanto à probabilidade de serem dependentes de álcool em relação ao grupo de referência (sem dependência / sem abuso) demonstrou que as variáveis com maior impacto foram sexo, exposição à violência parental e história de violência, nesta ordem. Assim, a probabilidade de dependência do álcool, quando comparada a sem dependência/sem abuso, foi 4,79 vezes maior (IC95\% $=[3,12 ; 7,35])$ em homens do que em mulheres, enquanto controlava as demais características.

Tabela - 4 Variáveis de maior impacto na determinação do uso nocivo ou dependência de álcool na idade adulta

\begin{tabular}{|c|c|c|c|c|c|}
\hline Características & Estimativa & $\begin{array}{c}\text { Erro } \\
\text { padrão }\end{array}$ & $\mathbf{t}$ & Valor de $p$ & $\begin{array}{r}\text { Odds } \\
\text { ratio }\end{array}$ \\
\hline \multicolumn{6}{|l|}{ Dependência de álcool } \\
\hline \multicolumn{6}{|l|}{ Sexo } \\
\hline Masculino & 1.566 & 0.217 & 7.210 & $<0.001$ & 4.79 \\
\hline Femino & - & & & & 1.00 \\
\hline \multicolumn{6}{|l|}{ Estado Civil } \\
\hline Casado & -0.578 & 0.171 & -3.370 & 0.001 & 0.56 \\
\hline Outros & - & & & & 1.00 \\
\hline \multicolumn{6}{|l|}{ Grupo étnico } \\
\hline Não Branca & 0.614 & 0.202 & 3.040 & 0.003 & 1.85 \\
\hline Branca & - & & & & 1.00 \\
\hline Exposição à Violência Parental & - & & & & 1.00 \\
\hline Exposição à violência severa & 0.675 & 0.214 & 3.150 & 0.002 & 1.96 \\
\hline Sem exposição/ Exposição à violência moderada & - & & & & 1.00 \\
\hline \multicolumn{6}{|l|}{ Abuso físico } \\
\hline Abuso Físico Severo & 0.647 & 0.257 & 2.520 & 0.013 & 1.91 \\
\hline Sem abuso/Abuso Físico Moderado & - & & & & 1.00 \\
\hline _cons & -3.655 & 0.268 & -13.650 & 0.000 & 0.03 \\
\hline \multicolumn{6}{|l|}{ Abuso de álcool } \\
\hline \multicolumn{6}{|l|}{ Sexo } \\
\hline Masculino & 1.799 & 0.311 & 5.790 & $<0.001$ & 6.04 \\
\hline Femino & - & & & & 1.00 \\
\hline \multicolumn{6}{|l|}{ Estado Civil } \\
\hline Casado & -0.105 & 0.290 & -0.360 & 0.717 & 0.90 \\
\hline Outros & - & & & & 1.00 \\
\hline \multicolumn{6}{|l|}{ Grupo étnico } \\
\hline Não Branca & 0.255 & 0.272 & 0.940 & 0.349 & 1.29 \\
\hline Branca & - & & & & 1.00 \\
\hline \multicolumn{6}{|l|}{ Exposição à Violência Parental } \\
\hline Exposição à violência severa & 0.187 & 0.413 & 0.450 & 0.651 & 1.21 \\
\hline Sem exposição/ Exposição à violência moderada & - & & & & 1.00 \\
\hline \multicolumn{6}{|l|}{ Abuso físico } \\
\hline Abuso Físico Severo & -0.321 & 0.502 & -0.640 & 0.523 & 0.73 \\
\hline Sem abuso/Abuso Físico Moderado & - & & & & 1,00 \\
\hline _cons & -4.673 & 0.390 & -11.970 & 0.000 & 0.01 \\
\hline
\end{tabular}


Além disso, o fato de que esta probabilidade foi 1,96 vezes maior $($ IC $95 \%=[1,29 ; 3,00])$ entre aqueles que foram expostos a violência parental grave do que outros que nunca tinham sido expostos a tal violência ou que só foram expostos a ameaças de violência parental na infância são dignas de nota.

$\mathrm{O}$ fato de ter sofrido abuso físico severo também apresentou probabilidade semelhante (1,91 - IC 95\% $=[1,15 ; 3,17])$, quando comparado àqueles que não sofreram abuso físico ou sofreram abuso físico moderado na infância. Além disso, a probabilidade de dependência do álcool (em relação ao sem dependência/sem abuso) foi $44 \%$ menor (odds ratio de 0,56 - IC95\% = [0,40; 0,79]) entre os indivíduos casados, quando comparados a outros grupos de estado civil. Por fim, essa probabilidade foi $85 \%$ maior (odds ratio de $1,85-\mathrm{IC} 95 \%=[1,24$; $2,75]$ ) entre brancos, quando comparados aos negros.

Nas análises da categoria abuso de álcool, nenhuma das variáveis (raça, estado civil, exposição e violência), alcançou um nível de significância de 5\%, exceto a variável sexo, na qual se observou que a probabilidade de abuso em homens é de cerca de seis vezes a da mulher $($ IC95\%=[3,27;11,17]).

\section{Discussão}

O presente estudo examinou a contribuição do abuso físico e da exposição à violência parental na infância para o desenvolvimento de desordens relacionadas ao uso de álcool (abuso e/ou dependência de álcool) subsequente na idade adulta em uma amostra nacionalmente representativa da população brasileira.

As prevalências de abuso físico e exposição à violência parental na infância encontradas foram semelhantes às informadas em pesquisas com amostras representativas da população geral de outros países ${ }^{(2-22)}$.

Os dois tipos de violência na infância avaliados pelo presente estudo foram significativamente associados entre si. Indivíduos com história de abuso físico apresentaram probabilidade 2,2 vezes maior de terem sido exposto à violência parental na infância de que a probabilidade daqueles que não relatam nenhum abuso físico (16,8\% dos sujeitos que não sofreram violência física foram expostos a violência parental na infância, enquanto $38 \%$ daqueles com histórico de violência foram expostos à violência parental na infância). Essas associações foram estatisticamente significativas para cada um dos tipos de violência estudados. Mais da metade dos participantes $(53,4 \%)$ sofreram abuso físico na infância ou foram expostos à violência dos pais. Assim, a dupla história de violência na infância, ou seja, abuso físico e exposição à violência parental, foi muito frequente na população estudada. Esses resultados corroboram outros estudos que relataram a coocorrência de ambos os tipos de violência ${ }^{(5,11,13)}$.

Com relação a desordens de uso de álcool, as taxas encontradas para abuso e/ou dependência de álcool estão em concordância com outros estudos brasileiros que utilizaram metodologias diferentes ${ }^{(23)}$.

Após ajuste para todas as variáveis do modelo, indivíduos com histórico de violência na infância, quando comparados àqueles sem história de violência na infância, apresentaram maiores probabilidades de sofrer abuso e/ou dependência de álcool na vida adulta. Mais especificamente, os indivíduos que foram expostos à violência parental na infância (controlados por variáveis sociodemográficas) revelaram 1,95 vezes mais probabilidade de apresentar dependência de álcool do que aqueles que não haviam sido expostos a esse tipo de violência.

Além disso, aqueles que sofreram abuso físico severo (controlado por variáveis sociodemográficas) tiveram quase 1,91 vezes mais chances de apresentar dependência de álcool, em comparação com aqueles que não foram abusados fisicamente. A forte relação entre história de violências e distúrbios relacionados ao uso subsequente de álcool em indivíduos da amostra foi semelhante à encontrada em outros estudos ${ }^{(8-9,24)}$.

Além disso, a análise de regressão multinomial constatou que, entre os homens, o abuso físico na infância foi o que mais afetou a probabilidade de desenvolver dependência de álcool, enquanto, entre as mulheres, foi a exposição à violência parental na infância. Assim, observando o efeito do abuso físico severo, nos homens, a probabilidade de dependência é cerca de duas vezes maior do que a daqueles que não sofreram nenhuma violência. Em contraste, o efeito da "exposição à violência parental moderada ou severa" na probabilidade de dependência de álcool em mulheres é cerca de três vezes maior do que a de outras pessoas que não foram expostas.

Dessa forma, os resultados do presente estudo confirmam que experiências de abuso físico na infância e exposição à violência parental entre pais são fatores de risco para o desenvolvimento de problemas subsequentes na vida, neste caso particular, problemas de uso de álcool.

Embora os resultados sugiram que o abuso físico na infância esteja associado a uma maior probabilidade de dependência de álcool na idade adulta, eles não esclarecem por que a violência sofrida na infância resultaria em efeitos adversos na vida adulta. Mais pesquisas são necessárias para determinar se existem diferenças nos efeitos do abuso físico e exposição à violência parental na infância sobre o desenvolvimento 
da dependência de álcool em mulheres e homens e como isso ocorre.

Para tanto, devem ser incluídos outros tipos de violência que possam coocorrer em famílias domesticamente violentas, como abuso psicológico, sexual e/ou negligência parental. Crescer em um ambiente violento pode resultar em exposições a outros riscos de saúde mental e física. Futuros estudos que investiguem esses caminhos potenciais em mais detalhes com dados longitudinais podem ser úteis, avaliando assim o impacto cumulativo de múltiplas exposições

O presente estudo tem algumas limitações que devem ser consideradas na interpretação dos resultados. Embora a relação causal entre história de violência na infância e transtornos por uso de álcool na idade adulta não possa ser determinada devido ao delineamento transversal deste estudo, foi encontrada uma associação entre esses dois eventos. Além disso, os dados são retrospectivos e baseados exclusivamente no autorrelato dos participantes, o que pode levar a um viés de memória ou relato

Apesar dessas limitações, este é o primeiro estudo que analisou a associação entre violência física e exposição à violência parental na infância e o desenvolvimento de transtornos relacionados ao uso de álcool na idade adulta com uma amostra representativa da população brasileira com 14 anos ou mais, de ambos os sexos e sem excluir nenhuma região brasileira (exceto aquelas mencionadas na seção de metodologia). A presente investigação seguiu os padrões de estudos probabilísticos internacionais, priorizando um rigor metodológico que resultou em dados de alta confiabilidade, confirmados pela ausência de viés de seleção, medidas adequadas aos objetivos propostos e intenso controle de qualidade em todas as etapas da pesquisa.

\section{Conclusão}

O presente estudo mostrou uma associação entre a ocorrência de abuso físico e a exposição à violência parental na infância, conforme relatado por adultos. Estudos futuros devem investigar porque certos sujeitos que vivenciam a violência na infância desenvolvem subsequentemente distúrbios do uso de álcool na vida, buscando identificar possíveis fatores de risco e proteção para a associação de tais eventos

Finalmente os resultados podem ter importantes implicações para a prevenção e intervenção, já que alertam os profissionais de saúde sobre a conexão entre a história de violência na infância e os transtornos por uso de álcool na vida adulta. Assim, tanto os pesquisadores quanto os médicos devem incluir o abuso físico e a exposição à violência parental na avaliação, uma vez que esses achados podem auxiliar na implementação de estratégias preventivas e programas de intervenção. voltados para a população com transtornos decorrentes do uso de álcool.

\section{Referências}

1. Zanoti-Jeronymo DV, Zaleski M, Pinsky I, Caetano R, Figlie NB, Laranjeira R. Prevalência de abuso físico na infância e exposição à violência parental em uma amostra brasileira. Cad Saúde Pública. 2009;25(11):2467-79.

2. Straus MA, Gelles RJ. Physical violence in American families: risk factors and adaptations to violence in 8145 families. New Brunswick, NJ: Transaction Books; 1995.

3. Briere J, Elliott DM. Prevalence and psychological sequelae of self-reported childhood physical and sexual abuse in a general population sample of men and women. Child Abuse Negl. 2003;27(10):1205-22.

4. Garcia-Moreno C, Jansen HA, Ellsberg M, Heise L, Watts $\mathrm{CH}$. Prevalence of intimate partner violence: findings from the WHO multi-country study on women's health and domestic violence. Lancet. 2006;368(9543):1260-9.

5. Dong M, Dube SR, Felitti VJ, Giles WH, Anda RF. Adverse childhood experiences and self-reported liver disease: New insights into the causal pathway. Arch Intern Med. 2003;163 (16):1949-56.

6. Sachs-Ericsson N, Blazer D, Plant EA, Arnow B. Childhood Sexual and Physical Abuse and the 1-Year Prevalence of Medical Problems in the National Comorbidity Survey. J Health Psychol. 2005;24(1):32-40.

7. MacMillan HL, Fleming JE, Streiner DL, Lin E, Boyle $\mathrm{MH}$, Jamieson $\mathrm{E}$, et al. Childhood abuse and lifetime psychopathology in a community sample. Am J Psychiatry. 2001; 158(11):1878-83.

8. Jasinski JL, Williams L M, Siegel J. Childhood physical and sexual abuse as risk factors for heavy drinking among African-American women: a prospective study. Child Abuse Negl. 2000; 24(8):1061-71.

9. Center for Substance Abuse Treatment. Substance Abuse Treatment for Persons With Child Abuse and Neglect Issues. Treatment Improvement Protocol 36. Rockville (MD): Substance Abuse and Mental Health Services Administration; 2000.

10. Cotton NS. The familial incidence of alcoholism: a review. J Stud Alcohol. 1979;40:89-116.

11. Caetano R, Field CA, Nelson S. Association Between Childhood Physical Abuse, Exposure to Parental Violence, and Alcohol Problems in Adulthood. J Interperl Violence. 2003;18(3):240-57. 
12. Dong M, Anda RF, Felitti VJ, Dube SR, Williamson DF, Thompson TJ, et al. The interrelatedness of multiple forms of childhood abuse, neglect, and household dysfunction. Child Abuse Negl. 2004;28(7):771-84.

13. Dube SR, Williamson DF, Thompson T, Felitti VJ, Anda RF. Assessing the reliability of retrospective reports of adverse childhood experiences among adult $\mathrm{HMO}$ members attending a primary care clinic. Child Abuse Negl. 2004; 28(7):729-37.

14. WHO. Global Status Report on Alcohol. Geneva: World Health Organization; 2004 [cited 2017 Jan 18]. Available from: http://www.who.int/substance_abuse/publications/ alcohol/en/index.html.

15. World Health Organization European Centre for Environment and Health. Alcohol and interpersonal violence: policy briefing. Rome: World Health Organization European Centre for Environment and Health; 2005 [cited 2017 Jan 18]. Available from: http://www.euro.who.int/_data/assets/ pdf_file/0004/98806/E87347.pdf.

16. Laranjeira R, Pinsky I, Zaleski M, Caetano R. I levantamento nacional sobre os padrões de consumo de álcool na população brasileira. Brasília, DF: Secretária Nacional Anti- Drogas - SENAD; 2007.

17. Caetano R, Ramisetty-Mikler S, Rodriguez LA. The Hispanic Americans Baseline Alcohol Survey (HABLAS): the association between birthplace, acculturation and alcohol abuse and dependence across Hispanic national groups. Drug Alcohol Depend. 2009;99(1-3):215-21.

18. Straus MA. Measuring intrafamily conflict and violence: the conflict tactics (CT) scales. In: Straus MA, Gelles RJ, editors. Physical violence in American families: risk factors and adaptations to violence in 8,125 families. New Brunswick, NJ: Transaction Books; 1990. p. 29-47.

19. Straus MA, Hamby SL, Boney-McCoy S, Sugarman DB. The Revised Conflict Tactics Scales (CTS2), development and preliminary psychometric data. J Fam Issues. 1996;17:283-316.

20. Straus M, Gelles RJ. Societal change and change in family violence from 1975 to 1985 as revealed by two national surveys. J Marriage Fam. 1986;48:465-79.

21. Cottler LB, Robins LN, Helzer JE. The reliability of the CIDISAM: a comprehensive substance abuse interview. Br J Addic. 1989;84(7):801-14.

22. Moore CG, Probst JC, Tompkins M, Cuffe S. Martin AB. The prevalence of violent disagreements in US families: effects of residence, race/ethnicity, and parental stress. Pediatrics. 2007;119(1S):S68-S76.

23. Carlini EA, Galduróz JCF, Noto AR, Carlini CM, Oliveira LG, Nappo AS, et al. II levantamento domiciliar sobre o uso de drogas psicotrópicas no Brasil: Estudo envolvendo as 108 maiores cidades do país 2005. [cited 2017 Feb 18]. Available from: http://www.unifesp.br/dpsicobio/cebrid/ lev_domiciliar2005/index.htm; 2007.
24. Rothman EF, Edwards EM, Heeren T, Hingson RW. Adverse Childhood Experiences Predict Earlier Age of Drinking Onset: Results From a Representative US Sample of Current or Former Drinkers. Pediatrics. 2008;122(2):e298-e304. 